\title{
Easy to pattern, chemically resistant 1-dimensional polymer photonic crystals
}

Mark Robertson, Ashley Sanford, Zhe Qiang*

School of Polymer Science and Engineering, The University of Southern Mississippi, 118 College Drive, \#5050, Hattiesburg, Mississippi 39406, USA

Corresponding to: Z.Q. (zhe.qiang@usm.edu)

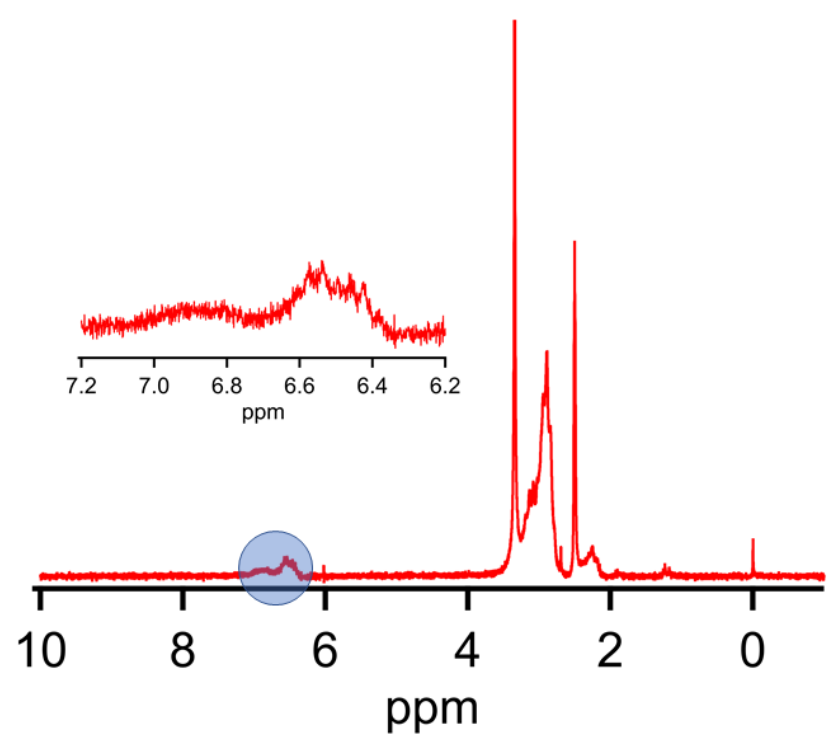

Figure S1. NMR spectrum of alkene-functionalized PVDF-CTFE. The peak from 6.4 to $7.0 \mathrm{ppm}$ represents the double bond that is formed as a result of the dehydrochlorination reaction. Degree of functionalization was calculated by integrating the peaks from $2.0-2.4 \mathrm{ppm}$ and $2.6-3.4 \mathrm{ppm}$ with respect to the alkene peak. 

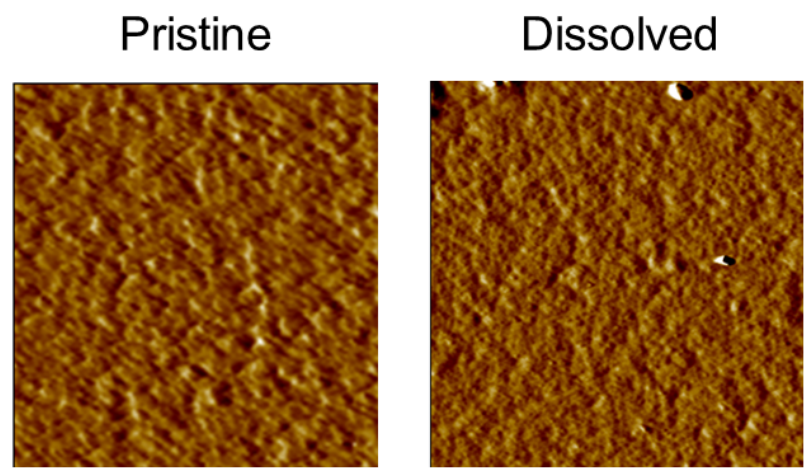

Figure S2. $10 \mu \mathrm{m}$ x $10 \mu \mathrm{m}$ AFM images of PVDF-CTFE before crosslinking and after crosslinking/removal of the soluble fraction of the film. While there is some increase in surface roughness, this result confirms that voids are not formed during processing.
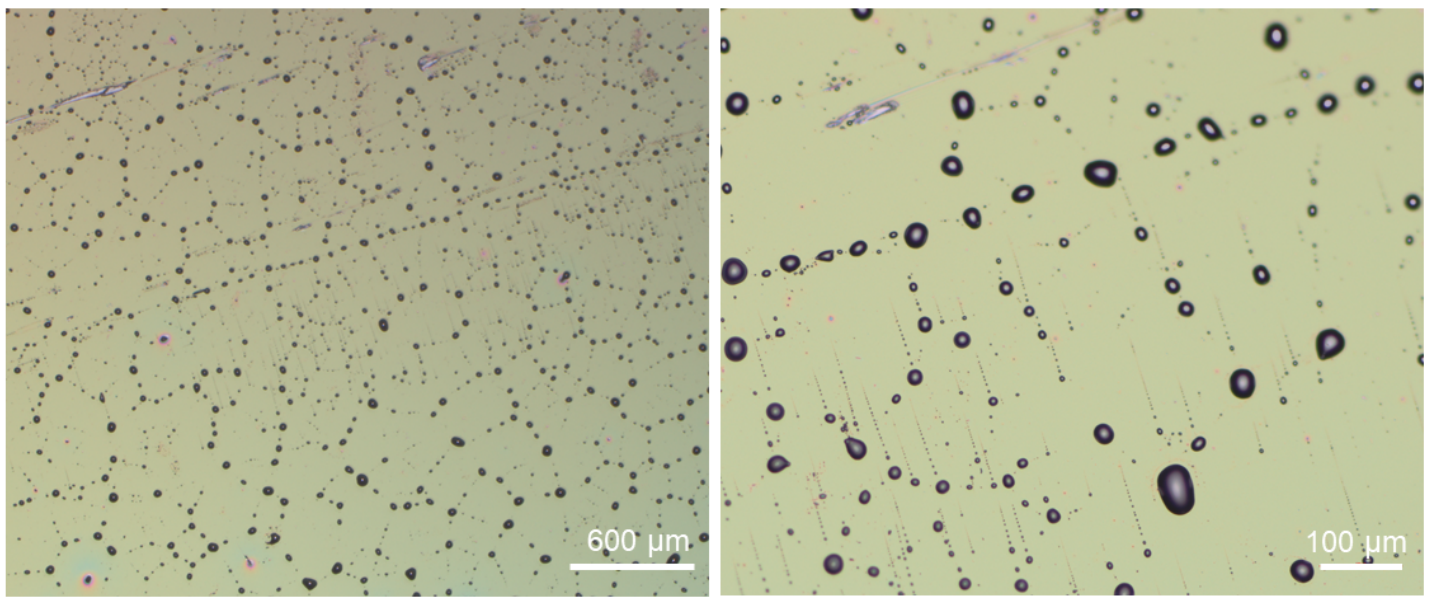

Figure S3. Optical images of dewet resol films as cast onto crosslinked PVDF-CTFE 


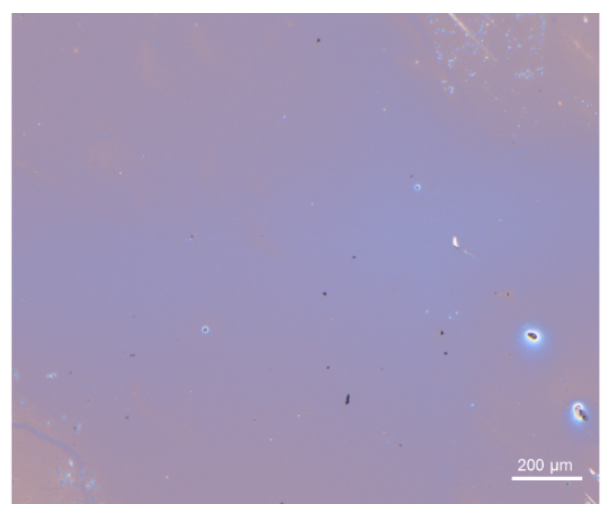

Figure S4. Optical image of successfully crosslinked resol cast onto crosslinked PVDF-CTFE after PVA adsorption.

\begin{tabular}{c|cccccc} 
& Sample 1 & Sample 2 & Sample 3 & Sample 4 & Sample 5 & Sample 6 \\
\hline $\begin{array}{c}\text { Predicted Reflectance Peak } \\
(\mathrm{nm})\end{array}$ & 410.4 & 427.8 & 467.4 & 527.8 & 587.8 & 619.8 \\
\hline Predicted PVDF-HFP film (nm) & 92.7 & 99.0 & 113.1 & 134.6 & 156.0 & 167.48 \\
\hline $\begin{array}{c}\text { Measured Reflectance Peak } \\
(\mathrm{nm})\end{array}$ & 379 & 480 & 409 & 538 & 464 & 608 \\
\hline Actual PVDF-HFP film (nm) & 86.2 & 106.8 & 96.9 & 143.0 & 116.8 & 168 \\
\hline
\end{tabular}

Table S1. Comparison of predicted reflectance peaks and PVDF-HFP film thicknesses against experimental values. 


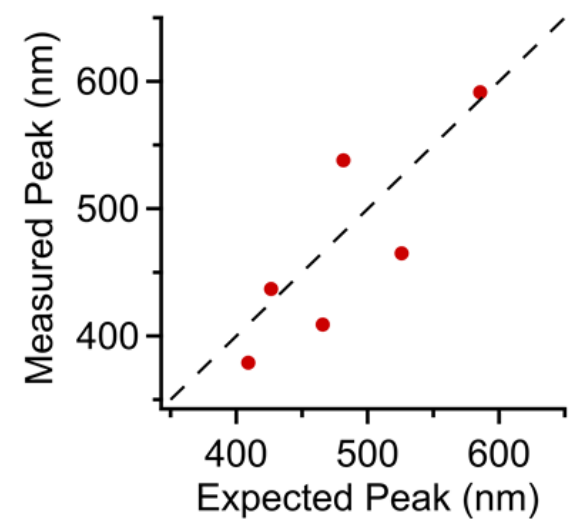

Figure S5. Relationship between the reflectance peaks from experiments and their expected peak position. A roughly $10 \%$ deviation was observed, which may be attributed to the variation in PVDF-CTFE film thickness as a result of trapped oxygen in the film. The equation we used to predict the peak wavelength takes the thickness shrinkage from soluble fraction after crosslinking into the consideration, expressed by:

$$
\lambda=2\left(1.40 * 0.49 \mathrm{~d}_{\text {(PVDF-CTFE) }}+1.64 * 46 \mathrm{~nm}\right),
$$

where 1.40 is the refractive index of PVDF-CTFE, $0.49 \mathrm{~d}_{\text {PVDF-CTFE }}$ is the thickness of PVDF-CTFE layer after crosslinking and the removal of soluble fraction, 1.64 and $46 \mathrm{~nm}$ are the refractive index and thickness of the deposited resol layers, respectively.

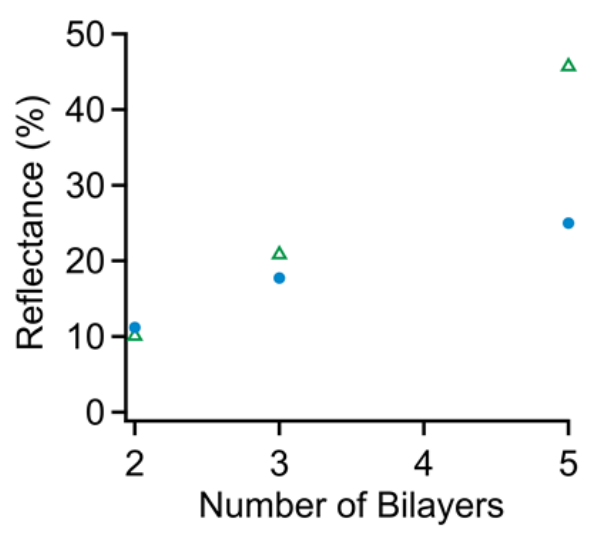

Figure S6. Relationship between reflectance and bilayers deposited onto a 1D-PC. The blue markers are experimentally verified values, and the green triangles represent theoretical values determined using Equation 2. 


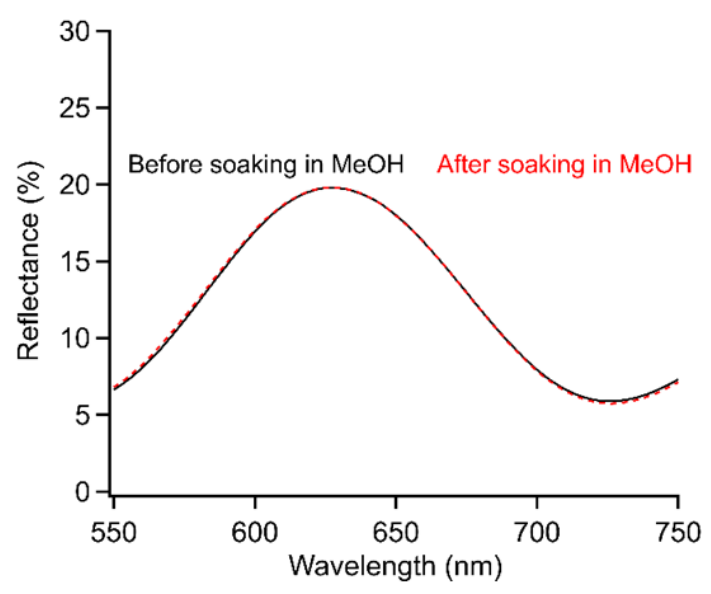

Figure S7. Reflectance spectra of a 1D-PC with a reflectance peak at $621 \mathrm{~nm}$ before and after direct immersion in methanol for two hours.

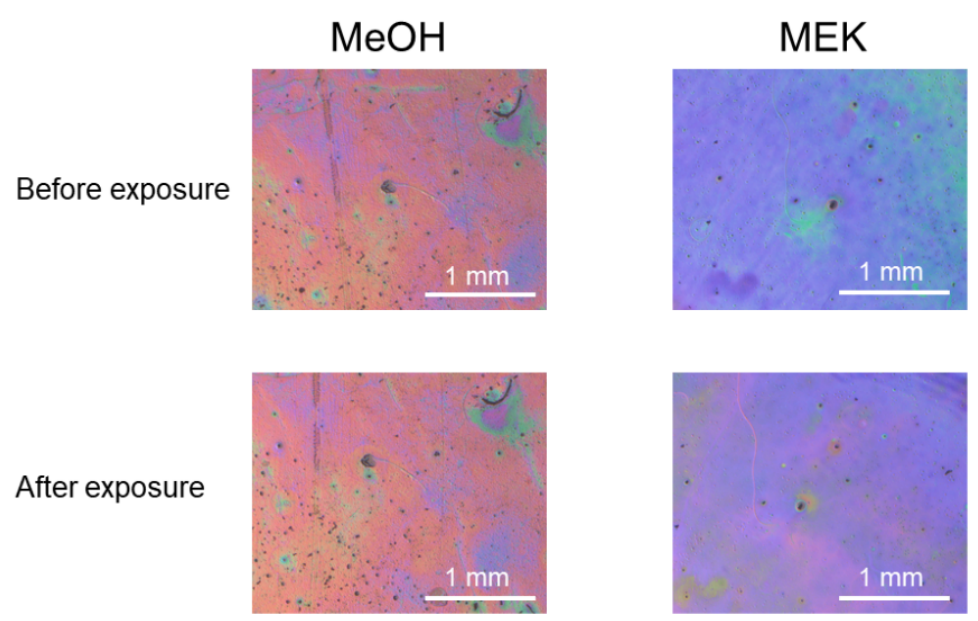

Figure S8. Optical images of 1D-PCs before and after exposure to methanol and MEK. 